\title{
Late complications in a man with poorly controlled congenital adrenal hyperplasia - case report
}

\author{
K. Maliszewska, A. Popławska- Kita, I. Kowalska, Maria Górska
}

Department of the Endocrinology, Diabetology and Internal Medicine, Medical University of Białystok,, Poland

\section{Introduction}

We report a case of $\mathrm{CAH}$ who developed several complications due to poor control. Proper diagnosis and treatment can enable men with the disease to have a normal life and fertility.

\section{Case report}

39-year-old patient with a history of primary adrenal insufficiency was admitted to our Department because of infertility. Semen analysis performed prior to hospitalization, showed azoospermia. The patient did not have medical documentation regarding his disease and was not able to explain exactly why the glucocorticoid therapy was initiated. In referral letter to the hospital, Addison's disease was written in the diagnosis. We learned from parents, that hydrocortisone was introduced after life-threatening episode in the neonatal period and maintained untill now. The study examination revealed dark complexion and brown spot on mucous membranes, livid lips, and enlarged testes with palpable nodules. Laboratory studies have shown: increased level of RBC, hyperlipidemia, high values of ACTH (>1250pg / ml) and testosterone (> $15 \mathrm{ng} / \mathrm{ml}$ ) and low concentrations of gonadotropins. After two days of hydrocortison withdrawal, cosyntropin test was performed revealing impaired cortisol response. Moreover, basal level of $27-\mathrm{OH}$ progesterone and after cosyntropin stimulation was very high. The abdominal computed tomography scan revealed grossly enlarged and heterogeneous adrenal glands (right $39 \times 71 \times 70 \mathrm{~mm}$, left $38 \times 89 \times 110 \mathrm{~mm})$. They showed progression comparing to previous CT. Ultrasonography of the testes revealed variable echogenicity. The tumor markers for germinative tumors (CEA, $\alpha-F P, \beta-H C G$ ) were negative. Biopsy of testes was performed. Urinary steroid profiling was performed to confirm the salt wasting form of $\mathrm{CAH}$. In the treatment $0,5 \mathrm{mg}$ of dexametasone was introduced.

\section{Laboratory results}

\begin{tabular}{|l|l|}
\hline & Laboratory results \\
\hline Morphology & $\mathrm{Ht}=57,4 \%, \mathrm{Hb}=19,0 \mathrm{~g} / \mathrm{dl}$ \\
\hline Testosteron & $>15 \mathrm{ng} / \mathrm{ml} ; \mathrm{N}: 1,56-8,77 \mathrm{ng} / \mathrm{ml}$ \\
\hline FSH & $0,16 \mathrm{mlU} / \mathrm{mL} ; \mathrm{N}: 1,37-13,5 \mathrm{mlU} / \mathrm{mL}$ \\
\hline LH & $0,16 \mathrm{mlU} / \mathrm{mL} ; \mathrm{N}: 1,37-13,5 \mathrm{mlU} / \mathrm{mL}$ \\
\hline ACTH & $>1250 \mathrm{pg} / \mathrm{ml} ; \mathrm{N}: 7,2-63,3 \mathrm{pg} / \mathrm{ml} ;$ \\
\hline $\begin{array}{l}17-O H \\
\text { progesteron }\end{array}$ & $>19,2 \mathrm{ng} / \mathrm{ml} ; \mathrm{N}: 0,2-1,3 \mathrm{ng} / \mathrm{ml} ;$ \\
\hline Na & $138 \mathrm{mmol} / \mathrm{l} ;$ \\
\hline K & $4,85 \mathrm{mmol} / \mathrm{l} ;$ \\
\hline Cholesterol & $231 \mathrm{mg} / \mathrm{d} ; \mathrm{N}<190 \mathrm{mg} / \mathrm{dl} ;$ \\
\hline Triglycerides & $286 \mathrm{mg} / \mathrm{dl} ; \mathrm{N}<150 \mathrm{mg} / \mathrm{dl}$ \\
\hline
\end{tabular}

\begin{tabular}{|c|c|c|}
\hline & Treatment & Results \\
\hline $\begin{array}{l}\text { At discharge from } \\
\text { hospital }\end{array}$ & $\begin{array}{l}0,5 \mathrm{mg} \\
\text { dexalmethasonum }\end{array}$ & $\begin{array}{l}\text { ACTH > } 1250 \mathrm{pg} / \mathrm{ml} \text {; } \\
\text { Testosteron > 15 } \\
\mathrm{ng} / \mathrm{ml} ; \\
\text { FSH: } 0,16 \mathrm{mlU} / \mathrm{mL} ; \\
\text { LH : } 0,03 \mathrm{mlU} / \mathrm{mL} ;\end{array}$ \\
\hline After 3 months & $\begin{array}{l}0,5 \mathrm{mg} \\
\text { dexamethasonum }\end{array}$ & $\begin{array}{l}\text { ACTH = 220pg/ml; } \\
\text { Testosteron = } \\
11.41 \mathrm{ng} / \mathrm{ml} ; \\
\text { FSH 1,33 LH = 0,2 } \\
\text { nlu } / \mathrm{ml} ;\end{array}$ \\
\hline
\end{tabular}

\section{Conclusions}

This case illustrates that congenital adrenal hyperplasia due to 21-hydroxylase deficiency can progress to chronic complications. Children with $\mathrm{CAH}$ require regular screening for complications and metabolic sequelae. It is desirable for all men with $\mathrm{CAH}$ to have a testicular ultrasound and routine semen analysis. Patients and their relatives should be well educated and informed about disease. 